\title{
Management of Type a Supracondylar Fractures Of Femur with Dynamic Condylar Screw (Des)
}

\author{
${ }^{1}$ Dr Sunil V. Patil,${ }^{2}$ Dr.P.B. Magdum, ${ }^{3}$ Dr. Nagesh P.Naik \\ ${ }^{1}$ Associate Professor, Department of Orthopaedics, Bharati Vidyapeeth Deemed University Medical College \\ and Hospital, Sangli \\ ${ }^{2,3}$ Professor, Department of Orthopaedics, Bharati Vidyapeeth Deemed University Medical College and \\ Hospital, Sangli
}

\begin{abstract}
Introduction: Supracondylar fractures of the femur in adults are relatively uncommon and account for only $7 \%$ of all femoral fractures ${ }^{l}$. However these fractures are often complex injuries, that present with numerous complications, like mal alignment, flexion contractures, stiffness and limb length discrepancies ${ }^{2}$ The purpose of this study was to assess the outcome of dynamic condylar screw in supracondylar fractures of femur in adults, while comparing the results with other international studies.

Material and methods:. This study was based on 30 patients of fresh supracondylar fractures of the femur treated with dynamic condylar screw at tertiary Trauma Center. The patients were followed up for 1 year and parameters like union, range of movements and time for healing were recorded. ${ }^{4}$ Schatzker and Lambert Criteria (Annexure I) were used to grade the results.

Results: The age range was from 18 years to 72 years. 18 patients were male and 12 were female. The cause of injury was high energy trauma like road traffic accidents and fall from height. All fractures were of AO type A. Based on above mentioned criteria, results were excellent in 18 patients (60\%), good in 6 patients (20\%), fair in 5 patients (17\%) and poor in 1 patient (3\%).

Conclusion: Dynamic condylar screw is one of the best devices to treat supracondylar fractures of the femur. Keywords: Supracondylar fracture femur, dynamic condylar screw. ,Primary Osteosynthesis.
\end{abstract}

\section{Introduction}

Supracondylar fractures of the femur although uncommon, are very challenging injuries to treat. These fractures occur in two different age groups - due to different types of injuries. In young patients these fractures occur due to high velocity injury e.g. Road traffic accidents, fire arm injuries and sport's injuries. While in elderly patients usually low velocity injury like fall during walking, results in supracondylar fractures of the femur. Treatment of these fractures have been a controversial subject over the past two decades. There have been a changing philosophy towards Surgical treatment of supracondylar fractures of femur ${ }^{1,2,3,4,5,}$.

Close management of these fractures was the treatment of choice until 1970. This was due to non availability of appropriate implants and lack of proper techniques. Apart from the usual problems of confining elderly patient to bed, conservative methods at any age may be complicated by knee stiffness, mal union and non union. Early surgical stabilization can facilitate care of the soft tissue, permit early mobility and reduces the complexity of nursing care ${ }^{6,7}$.

Open reduction and internal fixation has been advocated, using implants, including angle blade plate, fickle devices, Rush roads, Ender nails and interlocking nails. ${ }^{4,5,8,9,10}$. These implants can be used in various circumstances like simple fractures and complicated cases e.g. in fracture after TKA. But these devices are technically demanding and none of them can provide inter fragmental compression with good purchase in osteoporotic bones. However it is claimed that these problems can be solved with the use of dynamic condylar screw. Fixation of supracondylar fractures of femur with dynamic condylar screw has been advocated by various authors with satisfactory results ${ }^{11,12}$

\section{Material And Methods}

This study was conducted on 30 patients admitted in Orthopaedic unit of Bharati Medical College \& Hospital, Sangli, through emergency department from January 2005 to December 2008, having supracondylar fractures of the femur. AO classification was used to grade these fractures. Of 30 patients 15 were A1; 12 were A2 and 3 were A 3 type. Three patients were having grade I compound fractures which were debrided at time of admission after thorough Betadine, Hydrgen Peroxide \& saline wash.

Inclusion Criteria:

- AO type "A" supracondylar fracture.

- Both sexes 
- Age 18 years and above.

Exclusion Criteria:

1. Lower diaphyseal fractures of femur.

2. Pathological fractures.

3. AO type "B" and "C" fractures

4. Active infections any where in the body.

5. Medically unfit patients.

All the patients were evaluated for life threatening conditions as per ATLS protocol. Emergency treatment was provided in the accident and emergency department and after stabilization these patients were referred to the Orthopedic department for definitive management. Trauma surgery interval was 3-8 days with a mean of 4.2 days. After taking a proper history and meticulous physical examination the patients were prepared for the surgery. Templating on the antero-posterior and lateral views were done before the operation as pre-op preparations. The patients ware operated under Epidural anesthesia

Supine position with a pillow under knee was used. High tourniquet was applied . Standard lateral approach was taken and vastus lateralis was elevated from lateral intermuscular septum.. Dynamic condylar screw plate system was fixed as per standard method. Minimum four $4.5 \mathrm{~mm}$ cortical screws engaging 8 cortices were put in proximal fragment while two $6.5 \mathrm{~mm}$ cancellous screws were put in distal fragment in addition to condylar screw. In comminuted fractures the, area of comminution was exposed with care without much soft tissue dissection so that the fragments will maintain soft tissue attachment and biology will be maintained without going for anatomical reduction of fragments. Intravenous antibiotics were given for 5 days followed by oral antibiotics and analgesia. Patients were given continuous passive motion twice daily from third day with a aim of achieving at least 90 degree of movements at time of discharge that is tenth day. Patients were allowed only toe touch weight bearing for first six weeks During this period long knee brace. Patients were instructed to do knee bending; quadriceps and hamstring exercises at home. Partial weight bearing was started after reviewing $\mathrm{x}$ ray at six weeks. Full weight bearing was allowed at three months after reviewing union on $\mathrm{x}$ ray which was delayed if radiologically fracture was showing signs of delayed union. Clinical union was considered satisfactory if progressively increasing stiffness and strength provided by the mineralization process made the fracture site stable and pain free. Roentgenographic union was considered satisfactory when plain $\mathrm{x}$ rays showed bone trabeculae or cortical bone crossing the fracture site. Full weight bearing was allowed when radiological signs of union were present. ${ }^{\mathbf{4}}$ Schatzker and Lambert Criteria (Annexure I) were used to grade the results. Check $X$ rays were taken in immediate postoperative period, thereafter every six weeks up six months and then every three months interval up to one year. Final assessment of all the patients was done at one year. All the information regarding patient's age, sex, type of fracture, mechanism of injury, associated injuries, preoperative and postoperative complications, union time of fracture, partial weight bearing time of fracture, hospital stay were recorded on proforma specifically designed for this purpose. All the study variables were analyzed for descriptive statistics that is frequency and percentages.

\section{Observations And Results}

There were 18 males $(60 \%)$ and 12 females $(40 \%)$ with a male to female ratio of 3:2. The age range was from 18 years to 72 years with mean age of 42 years. 21 patients (70\%) were aged 25-50 years, 20\% were below 25 years and $10 \%$ above 50 years. Nineteen patients had fractures on right side and 11 patients on left side.

Average stay in hospital was 18.8 days; most patients quickly regained mobility after surgery. All patients achieved full extension The amount of extension achieved is shown in table I

Table 1: Range of Movements

\begin{tabular}{|l|l|l|l|}
\hline Sr No & Flexion of Knee Joint & No Of Patients. & Percentage \\
\hline 1 & More Than $120^{\circ}$ & 15 & $50 \%$ \\
\hline 2 & Between $90-120^{\circ}$ & 10 & $33.5 \%$ \\
\hline 3 & Less than $90^{\circ}$ & 05 & $16.5 \%$ \\
\hline
\end{tabular}

Clinical and radiological union was achieved in all patients. Details are shown in Table 2.

Table 2: Time for Radiological Union.

\begin{tabular}{|l|l|l|}
\hline Time for Radiological Healing & No Of Patients & Percentage \\
\hline $08-12$ weeks & 08 & $26.5 \%$ \\
\hline $12-16$ weeks & 21 & $70 \%$ \\
\hline More than 16 weeks & 01 & $3.5 \%$ \\
\hline
\end{tabular}

No patient had either mal union or nonunion of the fractures. 
Table No 3: Grading of the results using. ${ }^{4}$ Schatzker and Lambert Criteria

\begin{tabular}{|l|l|l|}
\hline Results at 1 year follow-up & No Of Patients & Percentage \\
\hline Excellent & 18 & $60 \%$ \\
\hline Good & 6 & $20 \%$ \\
\hline Fair & 5 & $17 \%$ \\
\hline Poor & 1 & $3 \%$ \\
\hline
\end{tabular}

\section{Clinical Cases..}

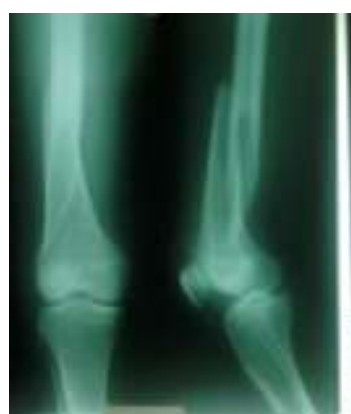

Fig1a

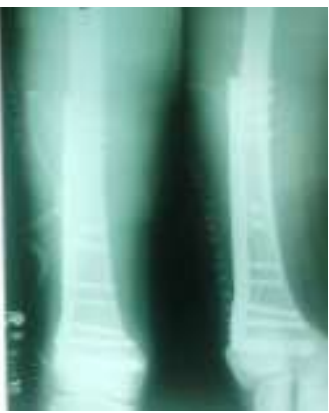

Xrays Excellent results. 4 months post-op.

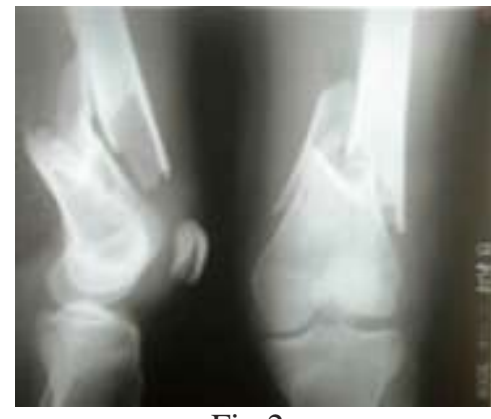

Fig 2a

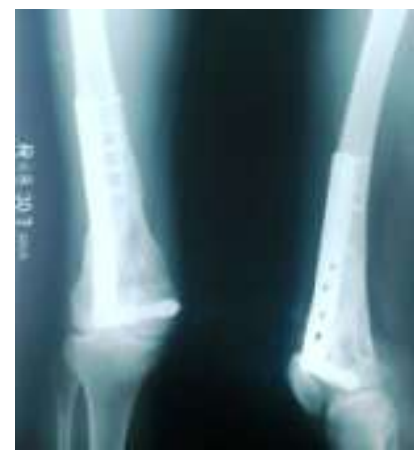

Fig $2 b$

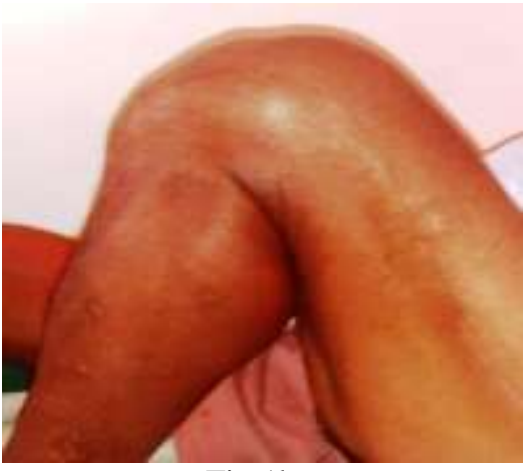

Fig $1 b$.

Fig 2a showing Communited Type A2 S/c Fracture

Fig $2 b$ showing xray of above Fracture Treated by DCS . \& Solid Union.4 months Post-op.

Fig 2c showing excellent clinical results.

\section{Complications:}

- One case had superficial infection which was treated with dressings and antibiotics No case had deep infection.

- One patient was lost in follow up and appeared after sex months with plate avulsed and broken screws. He was reoperated with the same procedure with addition of bone grafting and ultimately led to union after six more months.

- Three patients $(10 \%)$ had knee pain of moderate nature which responded well to oral analgesics. The cause of pain was degenerative osteoarthritis, due to old age. Five patients (16\%) were using walking stick as per instructions for osteoarthritis.

- Two of our patients $(7 \%)$ ended up with $2 \mathrm{~cm}$ shortening, which was managed with shoe raise. This shortening was intentional in both these cases, in order to get stable fixation in comminuted fractures.

- Long term results at 1 year follow up are shown in Table 3. 


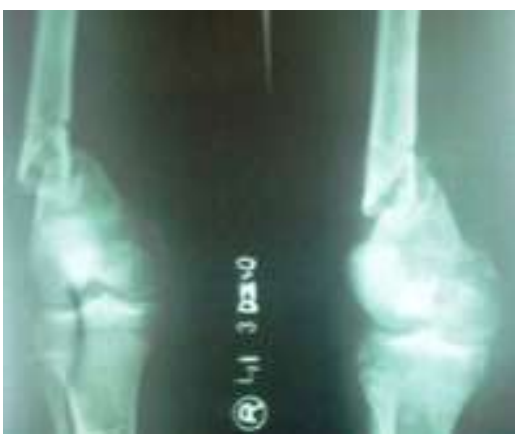

Fig 3a

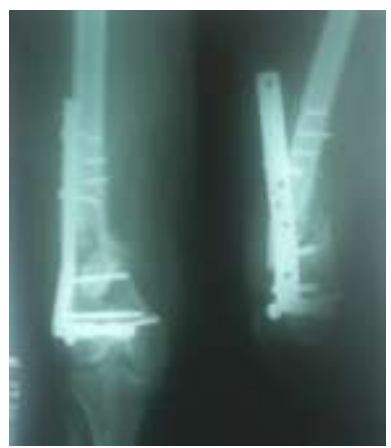

Fig3b

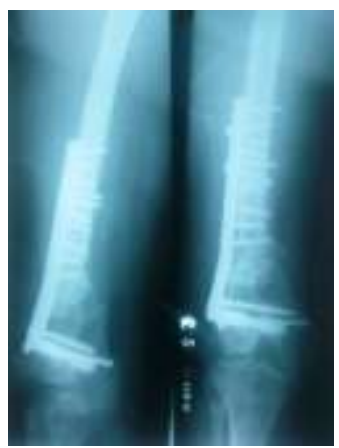

Fig 3c

Fig3a showing Communited A2 type S/C Frcature Femur.

Fig3b Showing above fracture fixed by DCS showing Breakage of Screws during Follow-up.

Fig3c Showing same fracture Fixed with DCS again with broken screws still inside.\& Bone Grafting was done .\& Xray showing Solid Union.

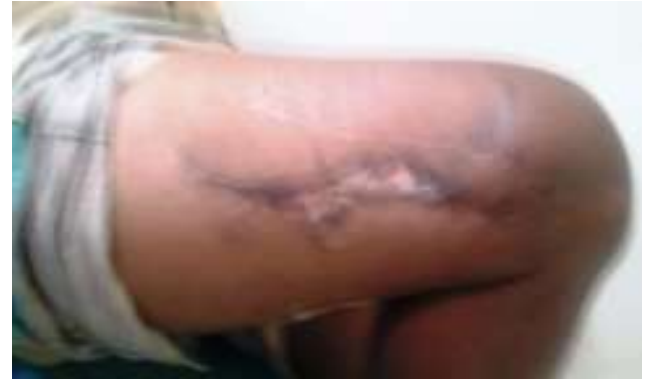

Fig3e

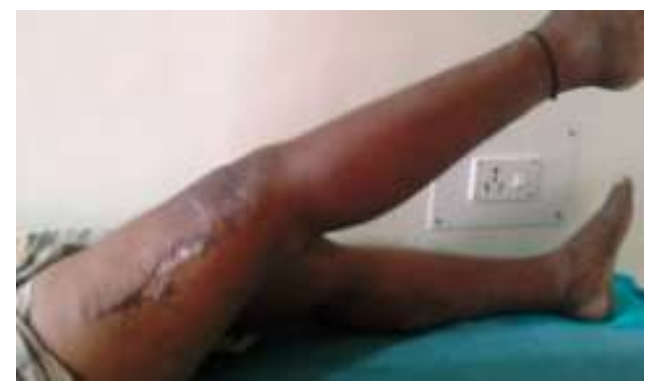

Fig3f

Fig 3e \& 3f showing good Clinical result of the same patient.

\section{Discussion}

Supracondylar fractures of the femur are often difficult to treat, and require careful management to obtain good cosmetic and functional results. It is difficult to counter-balance the powerful muscles forces around the fracture with close management. In recent years great advances have been made in the understanding and techniques of internal fixation. Over the time different types of implants have been used for the fixation of these fractures ${ }^{6,7}$. Dynamic condylar screw was found to be less technically demanding and provided good to excellent results as compared to other implants. ${ }^{13}$

In our study there was male preponderance due to more exposure of more males to trauma. Also working age group (30-50 years) people which are involved in outdoor activities got such fractures. As in this part of the country, .In Muslim countries the male to female ration is high as compared to western studies because of less active participation of females in outdoor activities in those societies ${ }^{14,15}$.

Road Traffic injuries are common, so this type of trauma accounted up to $33 \%$ of cases. KM Marya ${ }^{16}$ reported 92\% road traffic accidents and in Australian study ${ }^{17} 82 \%$ has been reported. Two patients had chest and abdominal injuries which were managed by concerned unit and then were shifted to orthopedic unit for management. Seven patients were having other orthopedic injuries. Twenty one patients had isolated lower limb injury. As place of present study is away from highway, the percentage of poly trauma patients is less as compared to German study ${ }^{18}$ where it amounted to $60 \%$

None of the patients were operated in first 24 hours as done in developed countries. ${ }^{19}$ This is because non availability of implants in emergency department, financial issues, and work load in orthopedic unit.

$80 \%$ of the patients in present series had excellent to good results Christodoulou et al ${ }^{15}$ reported excellent results in $51 \%$, good in $30 \%$, fair in $4 \%$, poor in $8 \%$ in total of 37 patients. which are comparable to present series.

Average time for union in present study was 15 weeks. Christodoulou et al ${ }^{15}$ reported time for union to be 20 weeks while a study at Addenbrook's hospital, Cambridge reported it to be 11.3 weeks. This vast difference in time taken for union in difference studies was due to differences in postoperative mobilizations protocols and criteria for union so cannot be compared to present series.

In 24 patients $(80 \%)$ full weight bearing was started at 12 weeks. In Addenbrook Hospital study ${ }^{20}$ showed better results with early weight bearing in third week of operation. Present study did not follow this protocol. 
Much attention was given to the range of knee movements in the follow up period. Initially passive movements for 2 days and then active movements were advised. Due to this follow up we obtained knee range of movement comparable to international studies ${ }^{1,2}$. In five patients the range of knee movements was less than $90^{\circ}$. These were the patients who were not attending the physiotherapy department and one of them was lost to follow up for a period of three months.

All fractures united ( one in second attempt) finally which is comparable to other national and international series. ${ }^{15}$ One case did not follow post operative protocol who came back after being lost in follow up with implant failure which needed refixation and bone grafting and finally achieved union. Huang Dong Fu et $^{\mathrm{al}^{21}}$ reported a nonunion rate of $6.94 \%$. Ayaz $\mathrm{M}$ et $\mathrm{al}^{22}$ study showed $0 \%$ nonunion rate.

Only one patient $(3.5 \%)$ had superficial infection. The reported rate is zero to eight percent in other studies which is comparable ${ }^{15,20}$.

\title{
V. Conclusion
}

The Dynamic condylar screw is an easy, less technically demanding and more rewarding method of treatment for a type of supracondylar fractures of the femur

\section{References}

[1] Giles JB., Delee JC., Heekman JD. Supracondylar -intercondylar fractures of the femur - treated with a supracondylar plate and lag - screw. JBJS, 1982, 64-A, 868.

[2] Healy WI., Brooker AF. Distal femur fractures: comparison of open and closed methods of treatment. Clin Orthop. 1983, $174,166$.

[3] Mise BD., Bnchol RW., Grogan DP. Surgical treatment of displaced, comminuted fractures of the distal end of the femur. JBJS. 1082, 64- A, 871.

[4] Schatzker J., Lambort DC. Supracondylar fractures of the femur. Clin Orthop. 1997, 138,77.

[5] Zickle RE., Fietti VG., Lawsing TF. A new intramedullary device for the distal third of the femur. Clin Orthop. $1997,125$.

[6] Engh GA., Ammeen DJ. Periprosthetic fractures adjacent to total knee implants. Treatment and clinical results JBJS 1992, 79, 1100-13.

[7] Pajarinen. LM. Pertroclanteric fractures of femur treated with dynamic limb screw J Bone Joint Surg (Br) 2005, 87-B, 76-81.

[8] Yang RS., Lin HC., Lin TK. Supracondylar femur fractures. J trauma 1990, 30, 315-9.

[9] Schelbourne KD., Buceckmann FR. Rush pin fixation of supracondylar and intercondylar femur fractures JBJS. 1982, 64-A, 161-9.

[10] Klomert L., Egind N., Person BM. Internal fixation of supracondylar and Bicondylar femur fractures using new semi elastic device. Clin Orthop. 1983, 204-19.

[11] Lumley JSP., Craver JC. Complications of fracture healing. Surgical International Vol : 54 . The medicine publishing company Ltd. West Sussex UK, 2001, 206.

[12] Pritchett JW. Supracondylar fractures of femur. Clin Orthop. 1984, 184, 173-7.

[13] Schewring DJ., Meggett BF. Fractures of the distal femur treated with A.O dynamic condylar screw JBJS. 1992, 74-B, 122-125.

[14] Iftikar Ali, Shahabuddin: Surgical outcome of supracondylar and intercondylar fractures femur in adults treated with dynamic condylar screw. JPMI2011 vol 2 no 1:49-55

[15] Christodoulou A. etal,Supracondylar fractures treated in elderly patients treated with dynamic condylar screw and retrograde intramedullary nail: a comparative study of the two methods. Arch Orthop TraumaSurg 2005;125:73-9

[16] Marya KM.Critical evaluation of management of fractures of shaft femur by Brooker- Willis nails. J Bone Joint Surg Am 2003;85:2093-96

[17] Majkowski RS, Baker As. Interlocking nails for femoral fractures an initial experienceInjury 1991;22:93-96

[18] Zhang BS, et al. Clinical study on rotational deformity after interlocked nailing in femur shaft fracture: Zhonghua Yi Xue Za Zhi 2005;85:2211-33

[19] Ozdemir HM etal. Immediate percutaneous fixation and functional bracing for the treatment of pediatric femoral shaft fracture J Pediar Orthop 2003;23:453-57

[20] Schwring DJ, et al.Fractures of the distal femur treated with the AO dynamic condylar screw. J Bone Joint Surg Br 1992;74:122-5

[21] Fu HD, et al Dynamic condylar screw for fracture of the distal femur. J Ortho Surg Taiwan 1996;3:237-42

\author{
Annexure I:- \\ Schatzker and Lambert Criteria3 \\ Excellent---full extension: \\ Flexion loss less than 10 degree \\ No varus, valgus or rotatory deformity \\ No pain \\ Perfect joint congruency \\ Good---not more than one of the following: \\ Loss of length not more than $1.2 \mathrm{~cm}$ \\ Less than 10 degree varus or valgus \\ Flexion loss not more than 20 degree \\ Minimal pain \\ Moderate---any two of the criteria in good category \\ Poor---any of the following: \\ Flexion to 90 degree or less \\ Varus or valgus deformity exceeding 15 degree \\ Joint incongruency \\ Disabling pain no matter how perfect the $\mathrm{x}$-ray
}

